\title{
Up-Regulation of the Vitamin D Pathway in Acute Myeloid Leukemia: A Novel Cause of Hypercalcemia
}

\author{
Casan J ML ${ }^{1 *}$, Ghotb $\mathrm{S}^{2}$, Morgan $\mathrm{S}^{1}$, Wei $\mathrm{AH}^{1,2}$ and \\ Ting SB ${ }^{2,3}$ \\ ${ }^{1}$ Department of Haematology, The Alfred Hospital, \\ Australia \\ ${ }^{2}$ Australian Centre for Blood Diseases, Monash \\ University, Australia \\ ${ }^{3}$ Department of Haematology, Eastern Health, Australia \\ *Correspondiing author: Casan J ML, Department \\ of Haematology, The Alfred Hospital, Commercial Rd, \\ Prahran 3181, Victoria, Australia
}

Received: February 06, 2017; Accepted: February 28, 2017; Published: March 13, 2017

\begin{abstract}
We report the first documented occurrence of hypercalcemia due to excessive production of calcitriol in acute myeloid leukemia (AML). The patient presented with severe hypercalcemia and renal failure accompanying a new diagnosis of AML. This extremely rare AML complication proved refractory to conventional hypercalcemia treatments but responded promptly to chemotherapy. Subsequent PCR studies performed on the leukemia blasts for vitamin D-related genes revealed dramatic up-regulation compared to healthy CD34+ cells and other primary AML cells. Recent data highlight a role for vitamin $D$ in hematopoietic cell proliferation. Accordingly, this case may highlight another aspect of molecular heterogeneity in AML.
\end{abstract}

Keywords: Acute leukemia; AML; Hypercalcemia; Pathology; Leukemia clinical

\section{Case Presentation}

Hypercalcemia frequently occurs as a paraneoplastic phenomenon, commonly accompanying solid organ cancers as well as lymphoid hematologic malignancies. However, hypercalcemia is a rare complication of myeloid cancer, and documentation in acute myeloid leukemia (AML) is limited to an exiguous collection of case reports [1-3]. The most commonly identified mechanisms of hypercalcemia in cancer include the secretion of parathyroid-related peptide (PTHrp), bony metastatic disease and tumour production of active vitamin D (calcitriol). The few published instances of hypercalcemia in AML have shown it to be secondary to either ectopic parathyroid hormone secretion, leukemic bony invasion or the release of bone resorptive mediators. We describe a case of newly diagnosed AML presenting with severe hypercalcemia causing acute kidney injury (AKI), subsequently demonstrated to be secondary to leukemic blast production of active vitamin D with gross overexpression of vitamin D-related genes, which resolved promptly following commencement of chemotherapy. Hypervitaminosis D is a well-documented cause of hypercalcemia and is known to occur in granulomatous diseases such as Sarcoidosis [4], as well as both Hodgkin and non-Hodgkin lymphomas [5]. However, to the best of our knowledge, there is no previous evidence of this phenomenon occurring in a myeloid malignancy. Accordingly, this case represents a novel pathogenic mechanism causing hypercalcemia in AML.

AA, a 68-year-old male presenting with fatigue and dyspnea was found to have circulating blasts on a full blood examination. The patient was referred to our institution and subsequently diagnosed with acute myelomonocytic leukemia (78\% blasts on bone marrow biopsy). Cytogenetic and fluorescence in-situ hybridisation demonstrated a normal karyotype, and flow cytometry revealed a myelomonocytic immunophenoytope with no aberrant lymphoid markers. Mutations in IDH2 (c.419G>A p.R140Q) and NPM1 (c.1165G >T) were detected by multiplexed mass spectrometry (MassARRAY System, Sequenom, San Diego, CA, USA). Accompanying the diagnosis of AML, AA had marked hypercalcemia (corrected calcium $3.3 \mathrm{mmol} / \mathrm{L}$, normal range 2.1-2.6 $\mathrm{mmol} / \mathrm{L}$ ) and AKI with serum creatinine measuring $263 \mathrm{umol} / \mathrm{L}$ (normal range $60-110 \mathrm{umol} / \mathrm{L}$ ). Given the rarity of AMLassociated hypercalcemia, we were reluctant to attribute this initially to his leukemia; therefore, extensive investigations were undertaken to elucidate the cause. In search of a second concurrent malignancy, the patient underwent computed tomography and positron emission tomography scanning, which revealed FDG avid vocal cord nodules. Direct laryngoscopy and multiple biopsies of these nodules revealed no malignant pathology. Parathyroid hormone (PTH) levels were appropriately suppressed $(0.9 \mathrm{pmol} / \mathrm{L}$, normal range $1.6-6.9 \mathrm{pmol} / \mathrm{L})$ and levels of PTHrp $(<2 \mathrm{pmol} / \mathrm{L})$ and serum ACE (37 units/L, normal range 20-70 units/L) were within normal limits. Inactive vitamin $\mathrm{D}$, (calcidiol or $25(\mathrm{OH}) \mathrm{D} 3)$ levels were also normal $(88 \mathrm{nmol} / \mathrm{L}$, normal range $>50 \mathrm{nmol} / \mathrm{L}$ ). However, the active vitamin $\mathrm{D}$ (calcitriol or $1,25(\mathrm{OH}) 2 \mathrm{D} 3)$ level was grossly elevated beyond the upper limit of the assay ( $>500 \mathrm{pmol} / \mathrm{L}$, upper limit of normal: $190 \mathrm{pmol} / \mathrm{L})$.

Both the hypercalcemia and kidney injury proved refractory to several initial therapeutic strategies including aggressive crystalloid hydration as well as an intravenous dose of $60 \mathrm{mg}$ of pamidronate. Such treatments would be expected to yield significant improvement in other conditions causing hypercalcemia. However, despite the failure of these first-line hypercalcemia therapies, there was a precipitous fall in calcium level following the initiation of chemotherapy with idarubicin and cytarabine, $7+3$ regimen (Figure 1). Within several days, AA's serum calcium levels returned to normal levels, and his kidney function followed a similar pattern of improvement, returning to his baseline level (90 umol/L) shortly thereafter. The rapid normalisation of serum calcium levels also mirrored peripheral blast clearance, and repeat testing of calcitriol levels over time has demonstrated progressive improvement towards a normal concentration. The patient achieved complete morphological remission with no detectable minimal residual disease (MRD) (10-colour flow cytometric method, sensitivity to $0.1 \%$ ) following induction chemotherapy. AA remains in complete remission with 


\section{Treatment Effect on Serum Calcium/Creatinine}

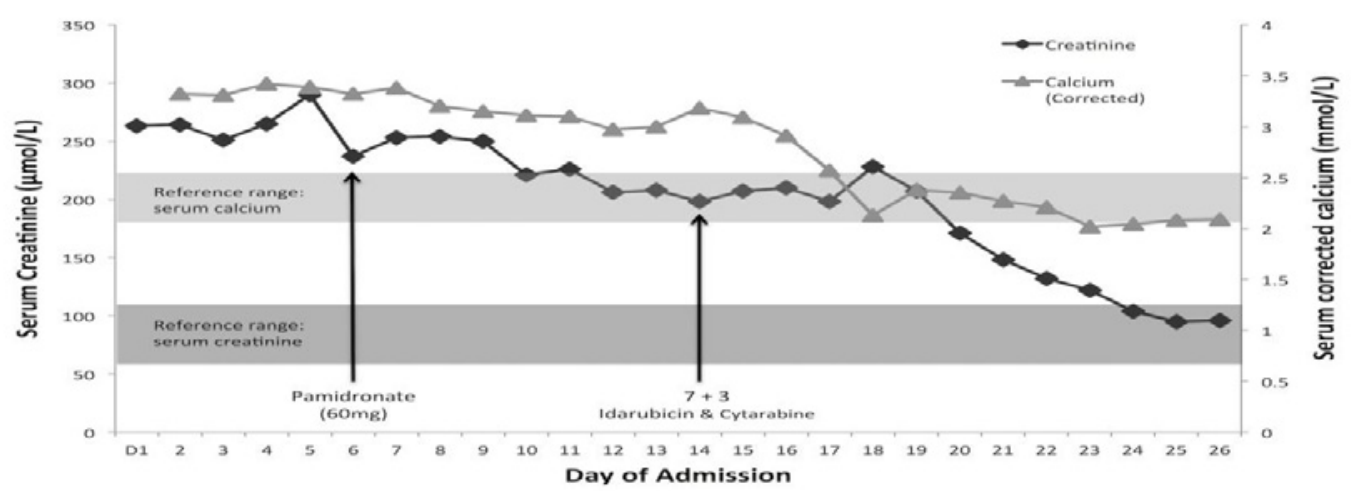

Figure 1: Serum calcium and creatinine across time and treatment. The patient's metabolic derangement proved only minimally responsive to conventional treatment, but normalised promptly after commencement of chemotherapy.

\section{RNA expression (Normalised to GAPDH)}

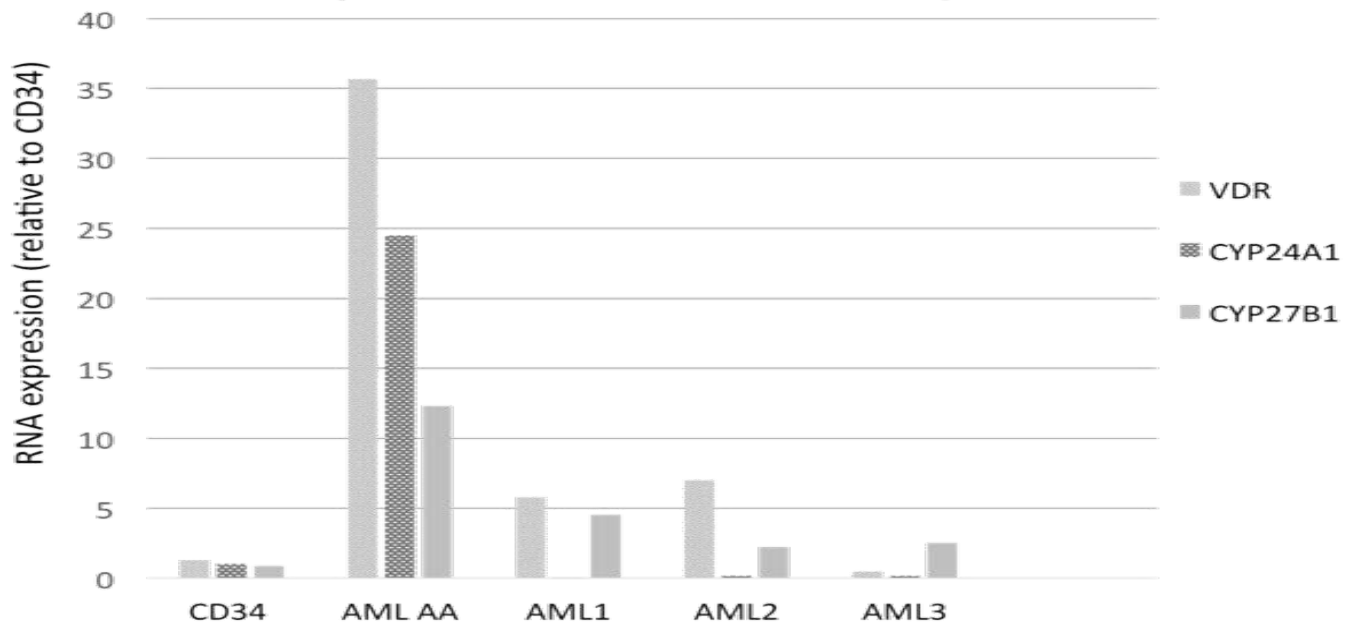

Figure 2: Vitamin D pathway genes in patient AA, normal CD34+ cells and 3 primary AML sample cells. AA's leukemic blasts showed significant up-regulation of all vitamin D-related genes compared with the other AML cells and healthy CD34+ cells.

MRD negativity after consolidation chemotherapy and is currently on maintenance azacitidine therapy. His hypercalcemia has not recurred and his renal function remains within normal limits and comparable to his baseline results.

Having excluded other causes of hypercalcemia, and given the dramatic improvement in response to chemotherapy, we hypothesised that the patient's AML blasts were synthesising calcitriol. Patient AA's diagnostic bone marrow was collected as part of our leukemia tissue bank repository, where the mononuclear cells are isolated using Ficoll preparation methodology and stored in TriZol. RNA was extracted from cells in TriZol using the QIAGEN RN easy kit and resuspended in $20 \mu \mathrm{l}$ of RNAase free water. RNA quantity and quality were assessed using the ND-1000 Spectrophotometer (Nanodrop). cDNA was synthesised from 400 ng of RNA using the Roche First Strand cDNA Synthesis Kit and C1000 ThermalCycler (Biorad). Quantitative real-time PCR ( $\mathrm{qPCR}$ ) of vitamin D genes relative to the housekeeping gene Gapdh was performed using the GoTaq qPCR reaction mix (Promega) and run on the LightCycler 480 II (Roche).

Using AA's diagnostic bone marrow mononuclear (leukemia) cell RNA, quantitative PCR was performed for genes essential to vitamin D metabolism: the vitamin-D receptor (VDR), CYP27B1 (1-a-hydroxylase, which converts calcidiol to calcitriol), and CYP24A1 (enzyme that hydroxylates calcitriol). Respective gene oligonucleotide primer sequences can be provided on request. These results were compared with healthy $\mathrm{CD} 34+$ cells and three other independent primary AML cells (Figure 2, labeled AML 1-3), which were selected for absence of respective patient hypercalcemia from our institution's tissue bank (Figure 2). These comparative AML samples originate from diagnostic bone marrow specimens processed as per patient AA's leukemia cells. AML1 was collected from a 24 year-old male with $93.8 \%$ blasts, trisomy 8 and ins(10;11); AML2 was collected from a 78 year-old male with $99 \%$ blasts, trisomy 13; 
AML3 was collected from a 36 year-old female with $80 \%$ blasts, $\mathrm{t}(9 ; 11)(9 \mathrm{q} 21,11 \mathrm{q} 22)$. None of these three comparative AML cells had detectable single gene molecular mutations.

\section{Discussion/Conclusion}

AA's leukemia cells demonstrated elevated expression of all the vitamin D-related genes compared to both healthy control CD34+ cells and the three other independent primary AML cells (Figure 2 ), suggesting the leukemic cells were capable of autonomously producing calcitriol and unaffected by physiologic negative feedback loops.

Hypercalcemia secondary to excessive calcitriol can be a manifestation of lymphoid malignancies; however, our case is the first documented occurrence of this phenomenon in a myeloid cancer. Our studies demonstrated striking over expression of vitamin D-related genes in leukemia cells, resulting in the patient's hypercalcemia and AKI. The hypercalcemia proved resistant to conventional treatments, including anti-osteoclast bisphosphonate therapy, which indicates a mechanism independent of potential direct leukemia-induced bone resorption. The near immediate response in both calcium and creatinine levels following the commencement of chemotherapy highlights this novel, leukemia cell-intrinsic mechanism of elevated calcitriol production leading to the rare complication of hypercalcemia in AML.

Study of a $V d r$-knockout mouse suggests a functional role for the vitamin D pathway in hematopoiesis, but possibly via a noncell autonomous mechanism [6]. Indeed, previous experiments have demonstrated that vitamin $\mathrm{D}$ treatment can induce monocytic differentiation of leukemic precursors [7]. Predicated on these preclinical data and the success of all-trans-retinoic acid differentiation therapy for acute promyelocytic leukemia (APML), non-APML AML cases have also been treated with vitamin D and analogues, but with limited success [8]. In the aforementioned context, it is not obviously clear why up-regulation of the vitamin $\mathrm{D}$ pathway would be advantageous to this patient's leukemic blasts. However, a recent publication intriguingly links vitamin $\mathrm{D}$ metabolism to teleost developmental hematopoiesis and human umbilical cord HSC proliferation [9]. In this study, zebrafish morpholino knockdown of essential vitamin D regulatory genes ( $v d r a$ and cyp27b1) was used to show that calcitriol could modify zebrafish hematopoietic stem progenitor cell production. In addition, exposing human CD34 ${ }^{+}$umbilical cord HSCs to calcitriol ex-vivo was shown to enhance proliferation, colony forming activity and cell survival [9]. Considering these findings, it is plausible that the patient's leukemic clone co-opted the vitamin D pathway to enhance leukemic cell proliferation without impacting on differentiation. Whether utilising vitamin $\mathrm{D}$ or its derivatives in the treatment of AML (as previously reported [8]) may in actuality, enhance leukemia cell proliferation in some AML cases is pure conjecture, but our case highlights that the molecular heterogeneity of AML can now also be extended to vitamin D metabolism.

\section{Declarations of Interest}

S.B.T is a recipient of an NHMRC Career Development Fellowship. There are no other declarations related to this work.

\section{Author Contributions}

J.M.L.C. and S.B.T. were the primary clinicians, designed the vitamin D related gene expression studies and wrote the paper; S.G. performed the qPCR experiments and generated Figure 2; S.M. was the primary pathologist and edited the paper; A.H.W. curates the tissue bank and edited the paper.

\section{References}

1. Gerwitz AM, Stewart AF, Vignery A, Hoffman R. Hypercalcemia complicating acute myelogenous leukaemia: a syndrome of multiple aetiologies. $\mathrm{Br} \mathrm{J}$ Haematology. 1983; 54: 133-141.

2. Zidar BL, Shadduck RK, Winkelstein A, Zeigler Z, Hawker CD. Acute myeloblastic leukemia and hypercalcemia: A case of probable ectopic parathyroid hormone production. N Eng J Med. 1976; 295: 692-694.

3. Muler JH, Valdez R, Hayes C, Kaminski M. Acute megakaryocytic leukemia presenting as hypercalcemia with skeletal lesions. Eur J Haematol. 2002; 68: 392-396.

4. Insogna KL, Dreyer BE, Mitnick M, Ellison AF, Broadus AE. Enhanced production rate of 1,25-dihydroxyvitamin $\mathrm{D}$ in sarcoidosis. J Clin Endocrinol Metab. 1988; 66: 72-75.

5. Seymour JF, Gagel RF. Calcitriol: the major humoral mediator of hypercalcemia in Hodgkin's disease and non-Hodgkin's lymphomas. Blood. 1993; 82: 1383-1394

6. Jeanson NT, Scadden DT. Vitamin D receptor deletion leads to increased hematopoietic stem and progenitor cells residing in the spleen. Blood. 2010; 116: $4126-4129$.

7. Marchwicka A, Cebrat M, Sampath P, Sniezewski L, Marcinkowska E. Perspectives of differentiation therapies of acute myeloid leukemia: the search for the molecular basis of patients' variable responses to 1,25-dihydroxyvitamin D and vitamin D analogs. Front Oncol. 2014; 4: 125.

8. Harrison JS, Bershadskiy A. Clinical experience using vitamin D and analogs in the treatment of myelodysplasia and acute myeloid leukemia: a review of the literature. Leuk Res Treat. 2012; 125814: 1-8.

9. Cortes M, Chen MJ, Stachura DL, Liu SY, Kwan W, Wright F, et al. Developmental vitamin D availability impacts hematopoietic stem cell production. Cell Rep. 2016; 17: 458-468.
Ann Hematol Oncol - Volume 4 Issue 3 - 2017
ISSN : 2375-7965 | www.austinpublishing group.com
Casan et al. @ All rights are reserved

Submit your Manuscript | www.austinpublishinggroup.com
Citation: Casan JML, Ghotb S, Morgan S, Wei AH and Ting SB. Up-Regulation of the Vitamin D Pathway in Acute Myeloid Leukemia: A Novel Cause of Hypercalcemia. Ann Hematol Oncol. 2017; 4(3): 1141. 